\title{
Guidelines in Implementing Enantioselective Indicator-Displacement Assays for $\alpha$-Hydroxylates and Diols
}

\author{
Lei Zhu, Zhenlin Zhong, and Eric V. Anslyn*
}

Department of Chemistry and Biochemistry, The University of Texas at Austin, Austin, TX 78712

\section{Supporting Information}

\section{Experimental Section}

General Methods. ${ }^{1} \mathrm{H}$ and ${ }^{13} \mathrm{C}$ NMR spectra were recorded on a Varian Unity Plus 300 spectrometer. The signals of the carbon atoms next to boron atoms were usually obscured because of C-B coupling. UV-vis measurements were performed on a Beckman DU-70 UV-vis spectrometer. Fluorescence spectra were measured on a PTI (Photon Technology International) Fluorimeter equipped with LPS-220B lamp power supply, a MD-5020 motor driver, and an 814 Photomultiplier Detection System. Low- and high-resolution mass spectra were recorded with Finnigan TSQ70 and VG Analytical ZAB2-E instruments, respectively. Reagents were used as purchased from various commercial sources. The protocol for receptor syntheses and determination of association constants between receptors and indicators or substrates via spectrophotometry were carried out as described elsewhere. ${ }^{28}$

7. ${ }^{1} \mathrm{H}$ NMR $\left(300 \mathrm{MHz}, \mathrm{CDCl}_{3}+1\right.$ drop $\left.\mathrm{CD}_{3} \mathrm{OD}\right) \delta 7.29(\mathrm{~d}, \mathrm{~J}=5.4 \mathrm{~Hz}, 1 \mathrm{H}), 7.04(\mathrm{~d}, \mathrm{~J}=5.1$ $\mathrm{Hz}, 1 \mathrm{H}), 3.84(\mathrm{~s}, 2 \mathrm{H}), 2.62(\mathrm{~m}, 6 \mathrm{H}), 1.84(\mathrm{~m}, 4 \mathrm{H}) ;{ }^{13} \mathrm{C} \mathrm{NMR}\left(75.5 \mathrm{MHz}, \mathrm{CDCl}_{3}+1\right.$ drop $\left.\mathrm{CD}_{3} \mathrm{OD}\right) \delta$ 134.8, 122.1, 54.2, 53.1, 23.6; HRMS (CI): calcd. $(\mathrm{M}+\mathrm{H})^{+} 212.0917$, found 212.0911.

8. ${ }^{1} \mathrm{H}$ NMR $\left(300 \mathrm{MHz}, \mathrm{CDCl}_{3}+1\right.$ drop $\left.\mathrm{CD}_{3} \mathrm{OD}\right) \delta 7.81(\mathrm{~m}, 1 \mathrm{H}), 7.28(\mathrm{~m}, 2 \mathrm{H}), 7.10(\mathrm{~m}, 1 \mathrm{H})$, $3.56(\mathrm{~s}, 2 \mathrm{H}), 2.69(\mathrm{~m}, 6 \mathrm{H}), 1.60(\mathrm{~s}(\mathrm{~b}), 6 \mathrm{H}) ;{ }^{13} \mathrm{C} \mathrm{NMR}\left(75.5 \mathrm{MHz}, \mathrm{CDCl}_{3}+1\right.$ drop $\left.\mathrm{CD}_{3} \mathrm{OD}\right) \delta$ 136.0, 129.8, 127.4, 64.6, 53.3, 25.3, 24.1; HRMS (CI): calcd. $(\mathrm{M}+\mathrm{H})^{+}$220.1509, found 220.1499 .

9. ${ }^{1} \mathrm{H}$ NMR $\left(300 \mathrm{MHz}, \mathrm{CDCl}_{3}+1\right.$ drop $\left.\mathrm{CD}_{3} \mathrm{OD}\right) \delta 7.29(\mathrm{~d}, \mathrm{~J}=5.1 \mathrm{~Hz}, 1 \mathrm{H}), 7.06(\mathrm{~d}, \mathrm{~J}=4.8$ $\mathrm{Hz}, 1 \mathrm{H}), 3.67(\mathrm{~s}, 2 \mathrm{H}), 2.81(\mathrm{~m}, 6 \mathrm{H}), 1.62(\mathrm{~m}, 6 \mathrm{H})$; HRMS $(\mathrm{CI})$ : calcd. $(\mathrm{M}+\mathrm{H})^{+} 226.1073$, found 226.1073 .

11. ${ }^{1} \mathrm{H}$ NMR $\left(300 \mathrm{MHz}, \mathrm{CDCl}_{3}+1\right.$ drop $\left.\mathrm{CD}_{3} \mathrm{OD}\right) \delta 7.88(\mathrm{~m}, 1 \mathrm{H}), 7.31-7.20(\mathrm{~m}, 3 \mathrm{H}), 4.04(\mathrm{~d}$, $\mathrm{J}=12.8 \mathrm{~Hz}, 1 \mathrm{H}), 3.48(\mathrm{~d}, \mathrm{~J}=12.8 \mathrm{~Hz}, 1 \mathrm{H}), 3.03(\mathrm{~m}, 2 \mathrm{H}), 2.07(\mathrm{~m}, 2 \mathrm{H}), 1.46(\mathrm{~m}, 2 \mathrm{H}), 1.05(\mathrm{~d}, \mathrm{~J}$ 
$=6.4 \mathrm{~Hz}, 6 \mathrm{H}) ;{ }^{13} \mathrm{C}$ NMR $\left(75.5 \mathrm{MHz}, \mathrm{CDCl}_{3}+1\right.$ drop $\left.\mathrm{CD}_{3} \mathrm{OD}\right) \delta 142.2,136.4,130.2,130.0$, 127.1, 55.6, 54.7, 30.5; HRMS (CI): calcd. $(\mathrm{M}+\mathrm{H})^{+} 234.1665$, found 234.1675.

12. ${ }^{1} \mathrm{H}$ NMR (300 MHz, $\mathrm{CDCl}_{3}+1$ drop $\left.\mathrm{CD}_{3} \mathrm{OD}\right) \delta 7.54(\mathrm{~m}, 1 \mathrm{H}), 7.28(\mathrm{~m}, 2 \mathrm{H}), 7.11(\mathrm{~m}, 1 \mathrm{H})$, $4.28(\mathrm{dd}, \mathrm{J}=15.0,18.3 \mathrm{~Hz}, 2 \mathrm{H}), 3.85(\mathrm{~m}, 1 \mathrm{H}), 3.73(\mathrm{dd}, \mathrm{J}=2.0,8.7 \mathrm{~Hz}, 1 \mathrm{H}), 3.32(\mathrm{~s}, 3 \mathrm{H}), 3.00$ (m, 1H), $2.83(\mathrm{~s}(\mathrm{~b}), 2 \mathrm{H}), 2.43(\mathrm{~m}, 1 \mathrm{H}), 2.27(\mathrm{~m}, 1 \mathrm{H}), 2.07(\mathrm{~m}, 2 \mathrm{H})$; MS (CI): calcd (M-OH) 246.13, $\left(2 \mathrm{M}-2 \mathrm{H}_{2} \mathrm{O}+\mathrm{H}\right)^{+}$491.38, found 246.0, 491.2.

13. ${ }^{1} \mathrm{H}$ NMR (300 MHz, $\mathrm{CDCl}_{3}+1$ drop $\left.\mathrm{CD}_{3} \mathrm{OD}\right) \delta 7.32(\mathrm{~d}, \mathrm{~J}=5.1 \mathrm{~Hz}, 1 \mathrm{H}), 7.06(\mathrm{~d}, \mathrm{~J}=5.1$ Hz, 1H), 4.19 (d, J = 13.3 Hz, 1H), 3.77 (d, J = 13.3 Hz, 1H), 3.67 (s, 3H), 3.34 (m, 1H), 3.08 $(\mathrm{m}, 1 \mathrm{H}), 2.50(\mathrm{~m}, 1 \mathrm{H}), 2.23(\mathrm{~m}, 1 \mathrm{H}), 2.08-1.88(\mathrm{~m}, 5 \mathrm{H}) ;{ }^{13} \mathrm{C} \mathrm{NMR}\left(75.5 \mathrm{MHz}, \mathrm{CDCl}_{3}+1\right.$ drop $\left.\mathrm{CD}_{3} \mathrm{OD}\right) \delta 173.3,146.8,135.0,122.4,65.2,52.8,52.7,52.2,29.5,22.9$; HRMS (CI): calcd. $(\mathrm{M}+\mathrm{H})^{+}$270.0971, found 270.0970 .

14. ${ }^{1} \mathrm{H}$ NMR (300 MHz, $\mathrm{CDCl}_{3}+1$ drop $\left.\mathrm{CD}_{3} \mathrm{OD}\right) \delta 7.20(\mathrm{~m}, 2 \mathrm{H}), 4.41(\mathrm{~m}, 1 \mathrm{H}), 4.28(\mathrm{~d}, \mathrm{~J}=12$ Hz, 1H), 4.03 (d, J = 12 Hz, 1H), 3.74 (t, J = 9.0 Hz, 1H), 3.68 (s, 3H), 3.31 (m, 1H), 2.62 (dd, J $=3.0,9.0 \mathrm{~Hz}, 1 \mathrm{H}), 2.20(\mathrm{~m}, 2 \mathrm{H})$.

16. ${ }^{1} \mathrm{H}$ NMR (300 MHz, $\mathrm{CDCl}_{3}+1$ drop $\left.\mathrm{CD}_{3} \mathrm{OD}\right) \delta 7.30(\mathrm{~d}, \mathrm{~J}=5.1 \mathrm{~Hz}, 1 \mathrm{H}), 7.05(\mathrm{~d}, \mathrm{~J}=4.9$ $\mathrm{Hz}, 1 \mathrm{H}), 4.39(\mathrm{~d}, \mathrm{~J}=13.8 \mathrm{~Hz}, 1 \mathrm{H}), 3.60(\mathrm{~d}, \mathrm{~J}=13.8 \mathrm{~Hz}, 1 \mathrm{H}), 3.51(\mathrm{dd}, \mathrm{J}=6.1,9.5 \mathrm{~Hz}, 1 \mathrm{H}), 3.40$ (dd, J = 5.4, $9.5 \mathrm{~Hz}, 1 \mathrm{H}), 3.33(\mathrm{~s}, 3 \mathrm{H}), 2.99(\mathrm{~m}, 1 \mathrm{H}), 2.82(\mathrm{~m}, 1 \mathrm{H}), 2.28(\mathrm{~m}, 1 \mathrm{H}), 2.03(\mathrm{~m}, 2 \mathrm{H})$, $1.76(\mathrm{~m}, 3 \mathrm{H}) ;{ }^{13} \mathrm{C}$ NMR $\left(75.5 \mathrm{MHz}, \mathrm{CDCl}_{3}+1\right.$ drop $\left.\mathrm{CD}_{3} \mathrm{OD}\right) \delta 148.1,134.9,122.0,75.5,64.0$, 59.2, 54.2, 28.5, 22.7; HRMS (CI): calcd. $(\mathrm{M}+\mathrm{H})^{+}$256.1179, found 256.1184 .

Determination of association constants $\left(K_{I}\right)$ between receptors and ML via fluorescence spectroscopy. A solution of $\mathrm{ML}(3.6 \mu \mathrm{M})$ and $8(79.8 \mu \mathrm{M})$ was titrated into a fluorescence cuvette containing solution of ML $(3.6 \mu \mathrm{M})$ at r.t. The sample was excited at $362 \mathrm{~nm}$ where $\mathrm{ML} / \mathbf{8}$ complex absorbed. The relative fluorescence intensity $\left(\mathrm{F} / \mathrm{F}_{\mathrm{I}}\right.$, Figure $\left.3 \mathrm{~B}\right)$ was plotted against the increasing total concentration of 8 at $446 \mathrm{~nm}$. The association constant $\left(\mathrm{K}_{\mathrm{I}}\right)$ was extracted through least squares regression curve-fitting of the data at $446 \mathrm{~nm}$ with the 1:1 binding isotherm equation (see supporting information) as a user-defined function in commercial software Origin.

Determination of association constants between receptors and $\alpha$-hydroxycarboxylates $\left(\mathbf{K}_{\mathbf{R}}, \mathbf{K}_{\mathbf{S}}\right)$ via fluorescence spectroscopy. A solution of ML (3.6 $\left.\mu \mathrm{M}\right),(\mathrm{S}, \mathrm{S})-\mathbf{1 0}(34 \mu \mathrm{M})$, and DPL $(3.0 \mathrm{mM})$ was titrated into a fluorescence cuvette containing a solution of ML $(3.6 \mu \mathrm{M})$ and 
(S,S)-10 (0.51 mM) at r.t. The sample was excited at $362 \mathrm{~nm}$ where the ML/(S,S)-10 complex absorbed. The fluorescence intensity at $446 \mathrm{~nm}$ was recorded after at least $1 \mathrm{~min}$ to allow the system to reach equilibrium. The association constant $\left(K_{R}\right)$ was determined with the competitive binding method, or the direct iterative curve fitting method developed in this study (Table 1).

Determination of $\mathbf{F} / \mathbf{F}_{\mathbf{I}}-\boldsymbol{e} \boldsymbol{e}$ correlation. Two ensemble solutions were prepared with ML (3.6 $\mu \mathrm{M}),(\mathrm{S}, \mathrm{S})-10(0.51 \mathrm{mM})$ and either D- (solution A) or L-PL (solution B) at $676 \mu \mathrm{M}$. Solution A was incrementally titrated into B until the ee reached 0, while the sample was excited at $362 \mathrm{~nm}$ and the fluorescence spectrum was recorded after at least $3 \mathrm{~min}$ to allow the system to reach equilibrium. The titration was repeated with $\mathrm{B}$ adding into A to complete the ee range from -1 to 1. $\mathrm{F}_{\mathrm{I}}$ was measured as the fluorescent intensity (CPS) of the free indicator ML $(3.6 \mu \mathrm{M})$ after the titration. Fluorescence intensity $\left(\mathrm{F} / \mathrm{F}_{\mathrm{I}}\right.$, Figure $\left.4 \mathrm{~B}\right)$ was plotted against $e e$ at $446 \mathrm{~nm}$, and the curve was iteratively fit with the program developed in this study (Figure 4B).

Derivation of eq. S09 used in iterative curve fitting of fluorescent 1:1 binding isotherms in Origin to determine association constants $\left(K_{I}\right)$ between receptors and 4-methylesculetin (ML).

$F=2.3 I_{0} \phi \varepsilon b c=k b c \quad\left(k \equiv 2.3 I_{0} \phi \varepsilon\right)$

$F=k_{H} b[H]+k_{I} b[I]+k_{H I} b[H I]$

When $k_{H}=0$ (presuming the receptor/host does not fluoresce),

$F=k_{I} b[I]+k_{H I} b[H I]$

When $H_{t}=0, F_{I}=k_{I} b[I]_{t}$.

$F / F_{I}=[I] /[I]_{t}+n[H I] /[I]_{t}\left(n \equiv k_{H I} / k_{I}\right)$

$[I]_{t}=[I]+[H I]$

$[H]_{t}=[H]+[H I]$

$[H I]=K_{I}[H][H I]$

variables: $F / F_{I},[\mathrm{I}],[\mathrm{HI}],[\mathrm{H}]$

From (S01) \& (S02):

$[I]=\left(n-F / F_{0}\right)[I]_{t} /(n-1)$

$[H I]=\left(F / F_{0}-1\right)[I]_{t} /(n-1)$ 


$$
\begin{aligned}
& {[H]=\frac{[H I]}{K_{I}[I]}=\frac{\left(F / F_{0}-1\right)}{K_{I}\left(n-F / F_{0}\right)}} \\
& {[H]_{t}=[H]+[H I]=\frac{\left(F / F_{0}-1\right)}{K_{I}\left(n-F / F_{0}\right)}+\frac{\left(F / F_{0}-1\right)[I]_{t}}{n-1}}
\end{aligned}
$$

From (S08):

$$
\begin{aligned}
& F / F_{0}=\left\{n-1+\left(n[H]_{t}+n[I]_{t}-[H]_{t}+[I]_{t}\right) K_{I}\right. \\
& \left.-(n-1) \sqrt{1+2[H]_{t} K_{I}+2[I]_{t} K_{I}+[H]_{t}^{2} K_{I}^{2}-2[H]_{t}[I]_{t} K_{I}^{2}+[I]_{t}^{2} K_{I}^{2}}\right\} /\left(2 K_{I}[I]_{t}\right)
\end{aligned}
$$

\section{Derivation of the competitive spectrophotometric method.}

All the terms in eq. S10 can be expressed in the terms of [H] (eqs S12 - S14) to afford eq. S15.

$$
[H]+[H I]+\left[H G_{R}\right]+\left[H G_{S}\right]=[H]_{t}
$$

From eqs 2 and 6 :

$$
\begin{aligned}
& {[I]=\frac{[I]_{t}}{1+K_{I}[H]}} \\
& {[H I]=\frac{K_{I}[I]_{t}[H]}{1+K_{I}[H]}}
\end{aligned}
$$

Eq.s (S13) and (S14):

$$
\begin{aligned}
{\left[H G_{R}\right] } & =\frac{\left(1+e e_{R}\right)[G]_{t}[H] K_{R}}{2\left(1+[H] K_{R}\right)} \\
{\left[H G_{S}\right] } & =\frac{\left(1-e e_{R}\right)[G]_{t}[H] K_{S}}{2\left(1+[H] K_{S}\right)}
\end{aligned}
$$

These equations are combined to give:

$$
[H]+\frac{K_{I}[I]_{t}[H]}{1+K_{I}[H]}+\frac{\left(1+e e_{R}\right)[G]_{t}[H] K_{R}}{2\left(1+[H] K_{R}\right)}+\frac{\left(1-e e_{R}\right)[G]_{t}[H] K_{S}}{2\left(1+[H] K_{S}\right)}=[H]_{t}
$$

Defining the indicator ratio $Q \equiv[I] /[H I]$ allows us to write $[H]=\frac{1}{Q K_{I}}$, which, when introduced into (S15), gives:

$$
\frac{1}{Q K_{I}}+\frac{[I]_{t}}{Q+1}+\frac{\left(1+e e_{R}\right)[G]_{t} K_{R}}{2\left(Q K_{I}+K_{R}\right)}+\frac{\left(1-e e_{R}\right)[G]_{t} K_{S}}{2\left(Q K_{I}+K_{S}\right)}=[H]_{t}
$$

If $K_{R}=K_{S} \equiv K, \mathrm{~S} 16$ can be simplified to: 
$[H]_{t}=\frac{1}{Q K_{I}}+\frac{[I]_{t}}{Q+1}+\frac{[G]_{t} K}{Q K_{I}+K}$

which is the typical equation for the competitive spectrophotometric method described in Connors.

\section{Iterative data fitting (A vs. ee)}

Equilibria:

$$
\begin{aligned}
& \mathrm{H}+\mathrm{I} \rightleftharpoons \mathrm{HI} \quad K_{\mathrm{I}}=[\mathrm{HI}] /([\mathrm{H}][\mathrm{I}]) \\
& \mathrm{H}+\mathrm{G}_{\mathrm{R}} \rightleftharpoons \mathrm{HG}_{\mathrm{R}} \quad K_{\mathrm{R}}=[\mathrm{HG}] /\left([\mathrm{H}]\left[\mathrm{G}_{\mathrm{R}}\right]\right) \\
& \mathrm{HI}+\mathrm{G}_{\mathrm{R}} \rightleftharpoons \mathrm{HG}_{\mathrm{R}}+\mathrm{I} \quad \Psi_{\mathrm{R}}=K_{\mathrm{R}} / K_{\mathrm{I}}=\left[\mathrm{HG}_{\mathrm{R}}\right][\mathrm{I}] /\left([\mathrm{HI}]\left[\mathrm{G}_{\mathrm{R}}\right]\right) \\
& \mathrm{H}+\mathrm{G}_{\mathrm{S}} \rightleftharpoons \mathrm{HG}_{\mathrm{S}} \quad K_{\mathrm{S}}=[\mathrm{HG}] /\left([\mathrm{H}]\left[\mathrm{G}_{\mathrm{S}}\right]\right) \\
& \mathrm{HI}+\mathrm{G}_{\mathrm{S}} \rightleftharpoons \mathrm{HG}_{\mathrm{S}}+\mathrm{I} \quad \Psi_{\mathrm{S}}=K_{\mathrm{S}} / K_{\mathrm{I}}=\left[\mathrm{HG}_{\mathrm{S}}\right][\mathrm{I}] /\left([\mathrm{HI}]\left[\mathrm{G}_{\mathrm{S}}\right]\right)
\end{aligned}
$$

According to the definition of $e e_{\mathrm{R}}$ (eq. 8),

$\left[\mathrm{G}_{\mathrm{R}}\right]_{\mathrm{t}}=\left[\mathrm{G}_{\mathrm{R}}\right]+\left[\mathrm{HG}_{\mathrm{R}}\right]=0.5\left(1+e e_{R}\right)[\mathrm{G}]_{\mathrm{t}}, \quad\left[\mathrm{G}_{\mathrm{S}}\right]_{\mathrm{t}}=\left[\mathrm{G}_{\mathrm{S}}\right]+\left[\mathrm{HG}_{\mathrm{S}}\right]=0.5\left(1-e e_{R}\right)[\mathrm{G}]_{\mathrm{t}}$

If $[\mathrm{H}]_{\mathrm{t}},[\mathrm{G}]_{\mathrm{t}},\left[\mathrm{G}_{\mathrm{R}}\right]_{\mathrm{t}},\left[\mathrm{G}_{\mathrm{S}}\right]_{\mathrm{t}}$, and $[\mathrm{I}]_{\mathrm{t}}$ are defined as the total concentrations of the host, guest, $R$-guest, $S$-guest, and indicator respectively, the following mass balance equations apply:

$[\mathrm{H}]_{\mathrm{t}}=[\mathrm{H}]+[\mathrm{HI}]+\left[\mathrm{HG}_{\mathrm{R}}\right]+\left[\mathrm{HG}_{\mathrm{S}}\right]$

$\left[\mathrm{G}_{\mathrm{R}}\right]_{\mathrm{t}}=\left[\mathrm{G}_{\mathrm{R}}\right]+\left[\mathrm{HG}_{\mathrm{R}}\right]$

$\left[\mathrm{G}_{\mathrm{S}}\right]_{\mathrm{t}}=\left[\mathrm{G}_{\mathrm{S}}\right]+\left[\mathrm{HG}_{\mathrm{S}}\right]$

$[\mathrm{I}]_{\mathrm{t}}=[\mathrm{I}]+[\mathrm{HI}]$

Substituting eq S24 and eq S26 into eq S20:

$$
\Psi_{\mathrm{R}}=\left[\mathrm{HG}_{\mathrm{R}}\right]\left(\mathrm{I}_{\mathrm{t}}-[\mathrm{HI}]\right) /\left([\mathrm{HI}]\left(\mathrm{G}_{\mathrm{Rt}}-\left[\mathrm{HG}_{\mathrm{R}}\right]\right)\right.
$$

$\left[\mathrm{HG}_{\mathrm{R}}\right]$ is solved from eq. S27:

$$
\left[\mathrm{HG}_{\mathrm{R}}\right]=\Psi_{\mathrm{R}}[\mathrm{HI}]\left[\mathrm{G}_{\mathrm{R}}\right]_{\mathrm{t}} /\left\{[\mathrm{I}]_{\mathrm{t}}+\left(\Psi_{\mathrm{R}}-1\right)[\mathrm{HI}]\right\}
$$

Similarly,

$$
\left[\mathrm{HG}_{\mathrm{S}}\right]=\Psi_{\mathrm{S}}[\mathrm{HI}]\left[\mathrm{G}_{\mathrm{S}}\right]_{\mathrm{t}} /\left\{[\mathrm{I}]_{\mathrm{t}}+\left(\Psi_{\mathrm{S}}-1\right)[\mathrm{HI}]\right\}
$$

Eq.s S18, S23, and S26 are combined to afford eq S30:

$$
K_{\mathrm{I}}=[\mathrm{HI}] /\left\{\left([\mathrm{H}]_{\mathrm{t}}-\left[\mathrm{HG}_{\mathrm{R}}\right]-\left[\mathrm{HG}_{\mathrm{s}}\right]-[\mathrm{HI}]\right)\left([\mathrm{I}]_{\mathrm{t}}-[\mathrm{HI}]\right)\right\}
$$

Introducing $\left[\mathrm{HG}_{\mathrm{R}}\right](\mathrm{S} 28)$ and $\left[\mathrm{HG}_{\mathrm{S}}\right](\mathrm{S} 29)$ into eq $\mathrm{S} 30$ and rearranging the resulted equation give a $4^{\text {th }}$ order polynomial equation $\mathrm{S} 31$ :

$$
\mathrm{a}[\mathrm{HI}]^{4}+\mathrm{b}[\mathrm{HI}]^{3}+\mathrm{c}[\mathrm{HI}]^{2}+\mathrm{d}[\mathrm{HI}]+\mathrm{e}=0
$$




$$
\begin{aligned}
& \text { where } \mathrm{a}=\mathrm{K}_{\mathrm{I}}\left(\Psi_{\mathrm{R}}-1\right)\left(\Psi_{\mathrm{S}}-1\right) ; \\
& \mathrm{b}=\Psi_{\mathrm{R}}-1-\left[\mathrm{G}_{\mathrm{R}}\right]_{\mathrm{t}} \mathrm{K}_{\mathrm{I}} \Psi_{\mathrm{R}}+\Psi_{\mathrm{S}}-\left[\mathrm{G}_{\mathrm{S}}\right]_{\mathrm{t}} \mathrm{K}_{\mathrm{I}} \Psi_{\mathrm{S}}-\Psi_{\mathrm{R}} \Psi_{\mathrm{S}}+\left[\mathrm{G}_{\mathrm{R}}\right]_{\mathrm{t}} \mathrm{K}_{\mathrm{I}} \Psi_{\mathrm{R}} \Psi_{\mathrm{S}}+ \\
& {\left[\mathrm{G}_{\mathrm{S}}\right]_{\mathrm{t}} \mathrm{K}_{\mathrm{I}} \Psi_{\mathrm{R}} \Psi_{\mathrm{S}}+[\mathrm{H}]_{\mathrm{t}} \mathrm{K}_{\mathrm{I}}\left(\Psi_{\mathrm{R}}+\Psi_{\mathrm{S}}-1-\Psi_{\mathrm{R}} \Psi_{\mathrm{S}}\right)+[\mathrm{I}]_{\mathrm{t}} \mathrm{K}_{\mathrm{I}}\left(2 \Psi_{\mathrm{R}}+2 \Psi_{\mathrm{S}}-3-\Psi_{\mathrm{R}} \Psi_{\mathrm{S}}\right) ;} \\
& \mathrm{c}=[\mathrm{I}]_{\mathrm{t}}\left[\Psi_{\mathrm{R}}-2-2\left[\mathrm{G}_{\mathrm{R}}\right]_{\mathrm{t}} \mathrm{K}_{\mathrm{I}} \Psi_{\mathrm{R}}+\Psi_{\mathrm{S}}-2\left[\mathrm{G}_{\mathrm{S}}\right]_{\mathrm{t}} \mathrm{K}_{\mathrm{I}} \Psi_{\mathrm{S}}+\left[\mathrm{G}_{\mathrm{R}}\right]_{\mathrm{t}} \mathrm{K}_{\mathrm{I}} \Psi_{\mathrm{R}} \Psi_{\mathrm{S}}+\right. \\
& \left.\left[\mathrm{G}_{\mathrm{S}}\right]_{\mathrm{t}} \mathrm{K}_{\mathrm{I}} \Psi_{\mathrm{R}} \Psi_{\mathrm{S}}+[\mathrm{I}]_{\mathrm{t}} \mathrm{K}_{\mathrm{I}}\left(\Psi_{\mathrm{R}}+\Psi_{\mathrm{S}}-3\right)+[\mathrm{H}]_{\mathrm{t}} \mathrm{K}_{\mathrm{I}}\left(2 \Psi_{\mathrm{R}}-\Psi_{\mathrm{R}} \Psi_{\mathrm{S}}-3+2 \Psi_{\mathrm{S}}\right)\right] \\
& \mathrm{d}=[\mathrm{I}]_{\mathrm{t}}^{2}\left[1+[\mathrm{I}]_{\mathrm{t}} \mathrm{K}_{\mathrm{I}}+\left[\mathrm{G}_{\mathrm{R}}\right]_{\mathrm{t}} \mathrm{K}_{\mathrm{I}} \Psi_{\mathrm{R}}+\left[\mathrm{G}_{\mathrm{S}}\right]_{\mathrm{t}} \mathrm{K}_{\mathrm{I}} \Psi_{\mathrm{S}}-[\mathrm{H}]_{\mathrm{t}} \mathrm{K}_{\mathrm{I}}\left(\Psi_{\mathrm{R}}+\Psi_{\mathrm{S}}-3\right)\right] \\
& \mathrm{e}=[\mathrm{H}]_{\mathrm{t}}[\mathrm{I}]_{\mathrm{t}}^{3} \mathrm{~K}_{\mathrm{I}}
\end{aligned}
$$

If the fluorescence of the host and guest molecules is negligible, the fluorescence of the system can be calculated as following:

$$
F / F_{\mathrm{I}}=[\mathrm{I}] /[\mathrm{I}]_{\mathrm{t}}+\mathrm{n}[\mathrm{HI}] /[\mathrm{I}]_{\mathrm{t}}=1+(\mathrm{n}-1)[\mathrm{HI}] /[\mathrm{I}]_{\mathrm{t}}
$$

$[\mathrm{HI}]$ is solved by Newton's method from eq S31 using $0.0001 \mathrm{I}_{\mathrm{t}}$ as an initial value. The result is used for the curve-fitting of eq S32. A short program is written in LabTalk as a user-defined function for the nonlinear least squares curve fitter of Origin.

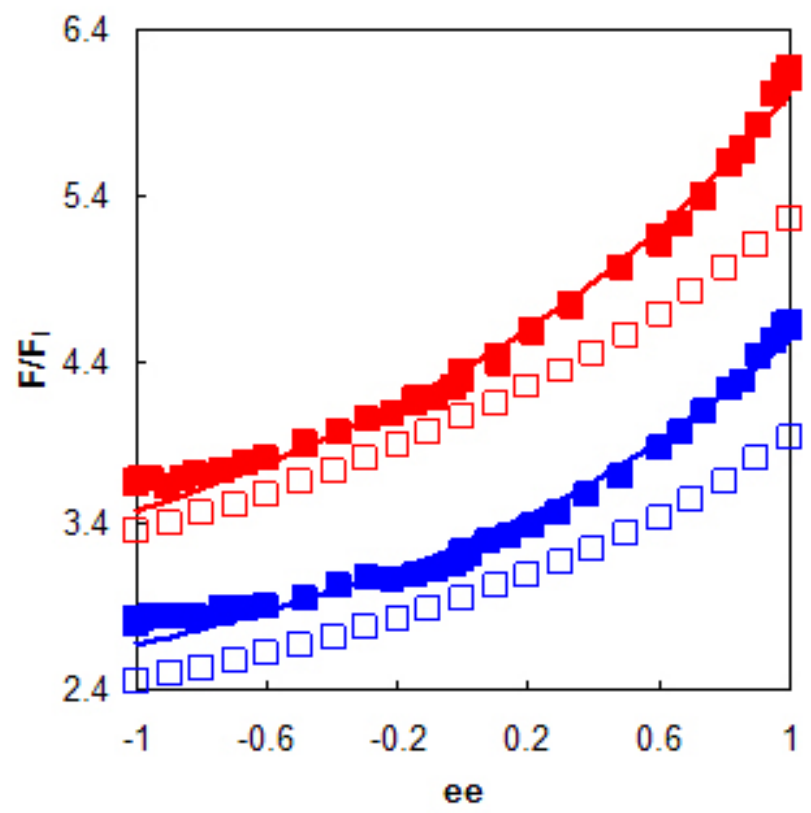

Figure 4B in the text. Empty squares are the data calculated with eq. 10 using parameters $\left(K_{R}\right.$ and $\mathrm{K}_{\mathrm{S}}$ ) determined individually, rather than determined from fitting of the $\mathrm{F} / \mathrm{F}_{\mathrm{I}} \mathrm{vs}$. ee curves. 